QCD Evolution Workshop 2014

International Journal of Modern Physics: Conference Series

Vol. 37 (2015) 1560021 (8 pages)

(C) The Author

DOI: $10.1142 / \mathrm{S} 2010194515600216$

\title{
Overview of TMD Factorization and Evolution
}

\author{
John Collins \\ 104 Davey Lab, Penn State University, \\ University Park PA 16802, USA \\ jcc8@psu.edu
}

Published 25 February 2015

\begin{abstract}
An overview is given of transverse-momentum-dependent (TMD) factorization and the associated evolution properties. Then a new method is presented for the schemeindependent comparison of different formalisms and fits.
\end{abstract}

\section{Introduction}

The aim of this talk was to give an overview of transverse-momentum-dependent (TMD) factorization and the associated evolution properties. I gave particular emphasis to properties of the non-perturbative part of the evolution kernel, since this has been particularly controversial in recent years. As a contribution to resolving the disagreements, I gave a first presentation of a new tool for the diagnosis and comparison of formalisms and fits. This uses a scheme-independent function $A\left(b_{\mathrm{T}}\right)$, which I will define; I show examples of its use. Throughout, I discussed the Drell-Yan (DY) process alone; however, the principles are general and apply equally to many other processes, such as were discussed at this workshop.

\section{Basics: Parton model to QCD}

A motivation for the idea of TMD factorization comes from the parton model, illustrated in Fig. 1. Two Lorentz contracted high-energy hadrons collide, and a quark and antiquark annihilate to the Drell-Yan pair (of invariant mass $Q$ ). The transverse momentum $\boldsymbol{q}_{\mathrm{T}}$ of the observed Drell-Yan pair is the sum of the transverse momenta of the annihilating partons. In the parton model, the partons are assumed to have distributions in longitudinal momentum fraction $x$ and transverse momentum $\boldsymbol{k}_{\mathrm{T}}$. The distribution of $\boldsymbol{q}_{\mathrm{T}}$ obeys Bjorken scaling, i.e., the distribution does not change when $Q$ (and hence the center-of-mass energy) is increased, with fixed parton momentum fractions.

This is an Open Access article published by World Scientific Publishing Company. It is distributed under the terms of the Creative Commons Attribution 3.0 (CC-BY) License. Further distribution of this work is permitted, provided the original work is properly cited. 


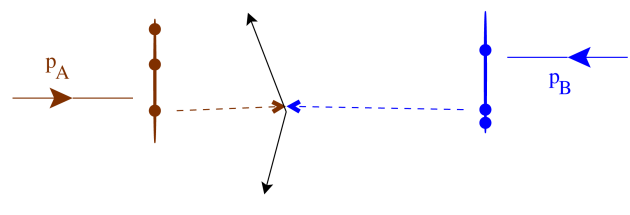

(a)

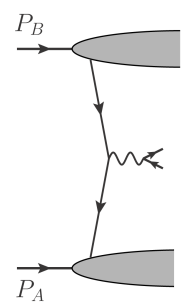

(b)

Fig. 1. (a) Spatial picture for parton model for Drell-Yan. (b) Amplitude implementing parton model.

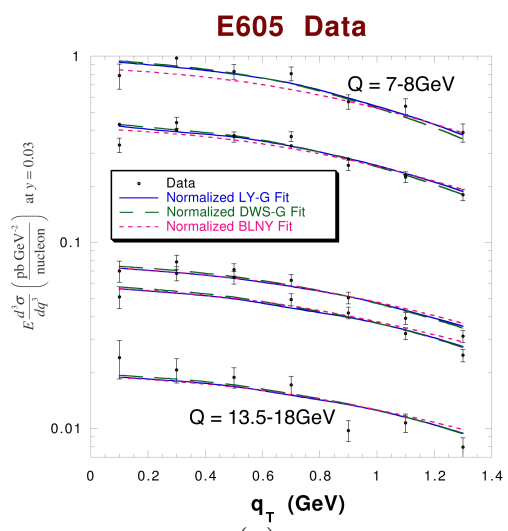

(a)

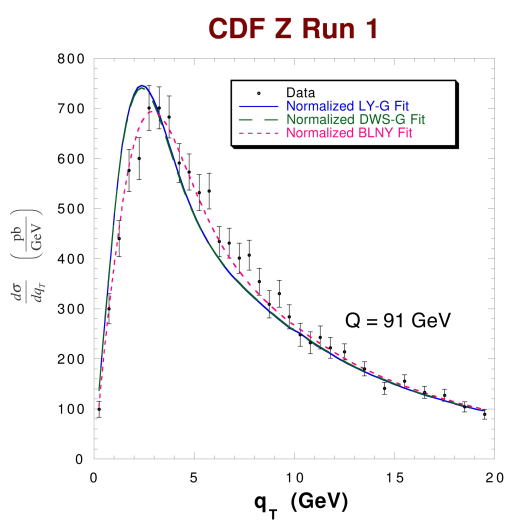

(b)

Fig. 2. The transverse-momentum distribution in the Drell-Yan process at different values of $Q$ and $\sqrt{s}$, showing data from the E605 and CDF experiments, together with some fits to the data using TMD factorization. (Adapted from plots by Landry et al. [1])

The parton model can be implemented in Feynman graph terms by assuming that graphs of the form of Fig. 1(b) dominate, with kinematics that match the parton-model intuition. However, the parton model needs to be substantially modified in QCD. This can be seen from data in Fig. 2. In graph (a) is shown data from E605 experiment at relatively low $Q=7-18 \mathrm{GeV}$ and $\sqrt{s}=38.8 \mathrm{GeV}$. In graph (b) is shown $\mathrm{d} \sigma / \mathrm{d} q_{\mathrm{T}}$ from the CDF experiment for $Z$ production at $\sqrt{s}=1800 \mathrm{GeV}$. Also shown are some fits to TMD factorization in QCD. The zero in graph (b) at $q_{\mathrm{T}}=0$, as compared with graph (a), is due to an extra factor of $q_{\mathrm{T}}$ in the definition of the cross section plotted, and does not represent any physical difference. The width in $q_{\mathrm{T}}$ for the high-energy data is much larger than for the lower energy data, and it also appears incompatible with any reasonable distribution of purely intrinsic transverse momentum. This implies that there are substantial evolution effects. (This implication is, in principle, subject to being confounded by the different parton $x$ for the two data sets.)

On the theory side, it is established from QCD, that the parton-model graphs and regions summarized in Fig. 1(b) are not the whole story. Much more complicated 


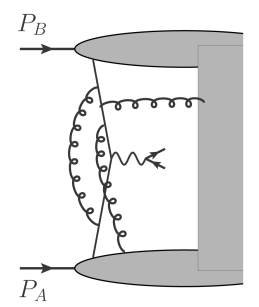

(a)

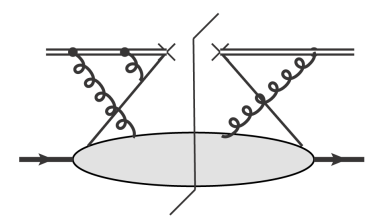

Fourier trans. of $\langle p| \bar{\psi}$ WL $\psi|p\rangle$

(b)

Fig. 3. Examples of: (a) leading QCD graphs for the Drell-Yan process, (b) graphs for parton density with Wilson line.

graphs, like those in Fig. 3(a), also contribute. To get to factorization, a substantial multistep derivation is needed - see Ref. [2] for a detailed account:

- Approximations are made for each relevant region at leading power.

- Ward identities are used to disentangle what one may call "misattached" gluons. This results in parton densities defined with Wilson lines in their operators, as in Fig. 3(b).

- Contour deformations and "unitarity cancellations", etc are needed.

- These results imply that the Wilson lines in the parton densities are initial-state Wilson lines, for DY.

- There can now be derived factorization of contributions of the different regions of loop momentum, obtained with the aid of appropriate subtractions.

- The definitions of the TMD pdfs are arranged so that the quanta inside are restricted to the appropriate hemisphere, etc. Soft gluons are absorbed into the modernized definitions.

A consequence of the graphs and the structure of the leading regions, is that there is broadening of transverse momentum distributions as energy and $Q$ are increased. This is caused by the emission of perturbative and non-perturbative glue into an increasing rapidity range.

In the form presented in Ref. [2] the TMD factorization formula for the DY cross section is

$$
\begin{aligned}
\frac{\mathrm{d} \sigma}{\mathrm{d}^{4} q \mathrm{~d} \Omega}= & \frac{2}{s} \sum_{j} \frac{\mathrm{d} \hat{\sigma}_{j \bar{\jmath}}(Q, \mu)}{\mathrm{d} \Omega} \int e^{i \boldsymbol{q}_{\mathrm{T}} \cdot \boldsymbol{b}_{\mathrm{T}}} \tilde{f}_{j / A}\left(x_{A}, \boldsymbol{b}_{\mathrm{T}} ; \zeta_{A}, \mu\right) \tilde{f}_{\bar{\jmath} / B}\left(x_{B}, \boldsymbol{b}_{\mathrm{T}} ; \zeta_{B}, \mu\right) \mathrm{d}^{2} \boldsymbol{b}_{\mathrm{T}} \\
& + \text { poln. terms }+ \text { high- } q_{\mathrm{T}} \text { term + power-suppressed. }
\end{aligned}
$$

Here, $\mathrm{d} \hat{\sigma}$ is the hard scattering coefficient, while the $\tilde{f}_{j / H}\left(x, \boldsymbol{b}_{\mathrm{T}} ; \zeta, \mu\right)$ are TMD parton densities Fourier transformed into transverse coordinate space. Their scale parameters can be set to $\zeta_{A}=\zeta_{B}=Q^{2}, \mu=Q$. The evolution equations have the form

$$
\frac{\partial \ln \tilde{f}_{j / H}\left(x, b_{\mathrm{T}} ; \zeta ; \mu\right)}{\partial \ln \sqrt{\zeta}}=\tilde{K}\left(b_{\mathrm{T}} ; \mu\right), \quad \frac{\mathrm{d} \tilde{K}}{\mathrm{~d} \ln \mu}=-\gamma_{K}\left(\alpha_{s}(\mu)\right)
$$




$$
\frac{\mathrm{d} \ln \tilde{f}_{j / H}\left(x, b_{\mathrm{T}} ; \zeta ; \mu\right)}{\mathrm{d} \ln \mu}=\gamma\left(\alpha_{s}(\mu) ; 1\right)-\frac{1}{2} \gamma_{K}\left(\alpha_{s}(\mu)\right) \ln \frac{\zeta}{\mu^{2}}
$$

Here, $\tilde{K}\left(b_{\mathrm{T}} ; \mu\right)$ is a defined function that controls the evolution of the TMD pdfs and fragmentation functions of light quarks with respect to the $\zeta$ parameter.

At small $b_{\mathrm{T}}$, there is a kind of operator-product expansion (OPE) that expresses the TMD density at small $b_{\mathrm{T}}$ in terms of the integrated densities:

$$
\tilde{f}_{j / H}\left(x, b_{\mathrm{T}} ; \zeta ; \mu\right)=\sum_{k} \int_{x-}^{1+} \tilde{C}_{j / k}\left(x / \hat{x}, b_{\mathrm{T}} ; \zeta, \mu, \alpha_{s}(\mu)\right) f_{k / H}(\hat{x} ; \mu) \frac{\mathrm{d} \hat{x}}{\hat{x}}+O\left[\left(m b_{\mathrm{T}}\right)^{p}\right] .
$$

The coefficients are perturbatively calculable provided that the TMD densities are evolved to scales that avoid large logarithms. The lowest-order value of the coefficients is $\delta_{j f} \delta(x / \hat{x}-1)$, which is the parton model result.

\section{Predictivity}

The key to predictivity of TMD factorization comes from a combination of universality of non-perturbative quantities, derived from QCD, and perturbative calculations:

- All process use the same TMD pdfs (and fragmentation functions),

- except that

- there is scale dependence governed by evolution equations,

- the Wilson lines in TMD pdfs are reversed in direction between DY and semiinclusive deeply inelastic scattering (SIDIS),

- which gives a sign reversal for the Sivers function etc.

- The same evolution kernel $\tilde{K}$ (color triplet) applies in all cases with quark-induced hard scattering, including all polarized cases (Sivers, Boer-Mulders, etc).

- (But note that there is a breakdown ${ }^{3}$ of TMD factorization in $H H \rightarrow H H+X$.)

The necessary non-perturbative information is found in

- the ordinary pdfs that appear in the OPE (4);

- the large $b_{\mathrm{T}}$ behavior of TMD pdfs (which can be labeled as equivalent to "intrinsic transverse momentum");

- the large $b_{\mathrm{T}}$ behavior of the evolution kernel $\tilde{K}\left(b_{\mathrm{T}}, \mu\right)$. This codes recoil against radiation per unit rapidity

Unfortunately, there is a variety of treatments, some of which disagree on the nature of the non-perturbative contributions at large $b_{\mathrm{T}}$, and even as to whether these non-perturbative contributions are needed. In their original work, Collins, Soper and Sterman $^{4}$ (CSS) took it as obvious that large $b_{\mathrm{T}}$ is non-perturbative. For work disputing the need for these non-perturbative contributions, see, for example, Refs. $[5,6,7,8]$. A summary of some of this and other work is in Ref. [9]. 


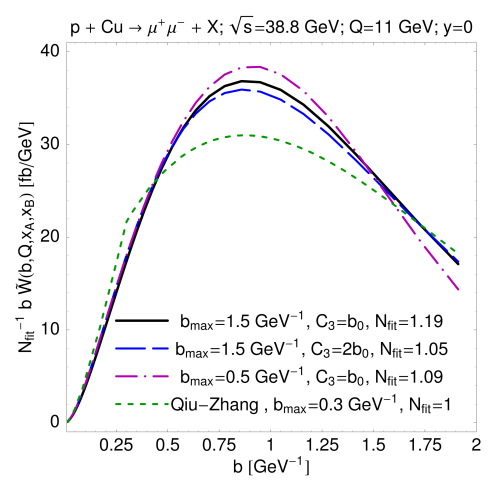

(a)

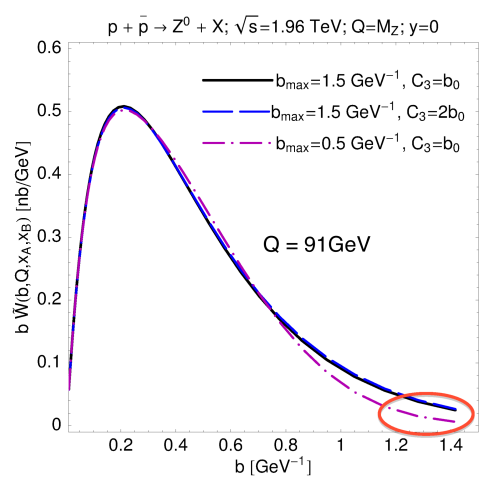

(b)

Fig. 4. Plots of the $b_{\mathrm{T}}$-space integrands corresponding to the cross section plots in Fig. 2, according to fits by Konychev and Nadolsky [10].

\section{Geography of TMD evolution; disagreements}

To see the nature of the issues involved, examine the plots in Fig. 4. Here are shown $b_{\mathrm{T}}$ integrands that correspond to the transverse-momentum cross sections in Fig. 2, as given by fits by Konychev and Nadolsky ${ }^{10}(\mathrm{KN})$. The result of evolution is to shift the important range of $b_{\mathrm{T}}$ to smaller values as energy increases. The $b_{*}$ method of CSS was used, which has a cut-off parameter $b_{\max }$ applied as a maximum distance to be treated in perturbative calculations (of $\tilde{K}\left(b_{\mathrm{T}}\right)$ etc). The primary difference we are concerned with here is between fits with $b_{\max }=0.5 \mathrm{GeV}^{-1}=$ $0.1 \mathrm{fm}$ and $b_{\max }=1.5 \mathrm{GeV}^{-1}=0.3 \mathrm{fm}$. In both cases, the non-perturbative part of $\tilde{K}\left(b_{\mathrm{T}}\right)$ was assumed to have quadratic dependence on $b_{\mathrm{T}}$. Good fits to the data were obtained, but the fit was significantly better with the larger, less conservative value of $b_{\max }=1.5 \mathrm{GeV}^{-1}$. In both cases $b_{\max }$ is less than the size of a proton.

The cross section is written as

$$
\frac{\mathrm{d} \sigma}{\mathrm{d}^{4} q}=\text { norm. } \times \int e^{i \boldsymbol{q}_{\mathrm{T}} \cdot \boldsymbol{b}_{\mathrm{T}}} \widetilde{W}\left(b_{\mathrm{T}}, s, x_{A}, x_{B}\right) \mathrm{d}^{2} \boldsymbol{b}_{\mathrm{T}} .
$$

Then in the CSS formulation, evolution of the integrand is governed by

$$
\left.\frac{\partial \widetilde{W}}{\partial \ln Q^{2}}\right|_{\text {fixed } x_{A}, x_{B}}=\left.\frac{\partial \widetilde{W}}{\partial \ln s}\right|_{\text {fixed } x_{A}, x_{B}}=\left(\tilde{K}\left(b_{\mathrm{T}}, \mu\right)+G(Q, \mu)\right) \widetilde{W},
$$

with $G$ being defined in terms of the hard scattering. The function $\tilde{K}\left(b_{\mathrm{T}}\right)$ is universal across all quark-induced processes. The function $G$ is perturbative, as is $\tilde{K}\left(b_{\mathrm{T}}\right)$ at small $b_{\mathrm{T}}$, provided that appropriate renormalization-group $(\mathrm{RG})$ improvement is applied. But $\tilde{K}\left(b_{\mathrm{T}}\right)$ at large $b_{\mathrm{T}}$ should be non-perturbative.

At the right-hand end of Fig. 4(b), for the high-energy data, I have ringed in red a region where there are factor of two differences between the two classes of fit. But this large difference has only a small effect on the cross section, because it is in a region where the integrand is small. The plots illustrate a general and well-known 


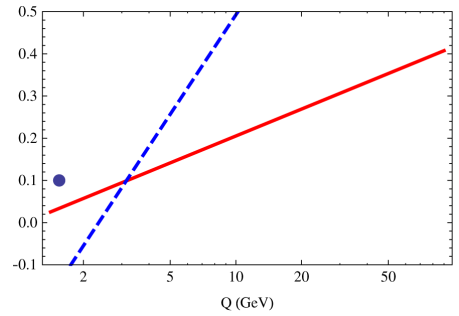

(a)

\begin{tabular}{ll}
$Q$ & Typical $b_{\mathrm{T}}$ \\
\hline $2 \mathrm{GeV}$ & $3 \mathrm{GeV}^{-1}$ \\
$10 \mathrm{GeV}$ & $1.2 \mathrm{GeV}^{-1}$ \\
$m_{Z}$ & $0.5 \mathrm{GeV}^{-1}$
\end{tabular}

(b)

Fig. 5. (a) Coefficient of $-b_{\mathrm{T}}^{2}$ in the exponent in Eq. (9), from Sun and Yuan [6], as a function of $Q$ at $x=0.1$. The blue dashed line is for the BLNY fit, and the red solid line for a KN fit with $b_{\max }=1.5 \mathrm{GeV}^{-1}$. The dot gives the value needed for SIDIS at HERMES. (b) Rough estimates of typical values of $b_{\mathrm{T}}$ at different $Q$.

phenomenon: As $Q$ is increased, the quantitative influence of the non-perturbative region is reduced, leading to dominance by perturbatively calculable $b_{\mathrm{T}}$ dependence, provided only that the non-perturbative part decays appropriately.

In contrast, sensitivity to the large- $b_{\mathrm{T}}$ region is enhanced at lower $Q$. In the $b_{*}$ prescription of CSS, one writes $\tilde{K}\left(b_{\mathrm{T}}, \mu\right)=\tilde{K}\left(b_{*}, \mu\right)-g_{K}\left(b_{\mathrm{T}} ; b_{\max }\right)$ Two standard fits at $Q$ up to $m_{Z}$ give:

$$
\begin{array}{ll}
g_{K}\left(b_{\mathrm{T}}\right)=\frac{0.68_{-0.02}^{+0.01} b_{\mathrm{T}}^{2}}{2} & \left(\mathrm{BLNY},{ }^{1} b_{\max }=0.5 \mathrm{GeV}^{-1}=0.1 \mathrm{fm}\right), \\
g_{K}\left(b_{\mathrm{T}}\right)=\frac{0.158 \pm 0.023}{2} b_{\mathrm{T}}^{2} & \left(\mathrm{KN},{ }^{10} b_{\max }=1.5 \mathrm{GeV}^{-1}=0.3 \mathrm{fm}\right) .
\end{array}
$$

(The BLNY fit is essentially the same as the $\mathrm{KN}$ fit with $b_{\max }=0.5 \mathrm{GeV}^{-1}$ used in Fig. 4.) But the quadratic fits give wrong behavior at lower $Q$, as Sun and Yuan 6 showed. With these parameterizations, the integrand is Gaussian at large $b_{\mathrm{T}}$ :

$$
\tilde{W}=\ldots e^{-b^{2}\left[\operatorname{coeff}(x)+\text { const } \ln \left(Q^{2} / Q_{0}^{2}\right)\right]}
$$

The coefficient of $-b_{\mathrm{T}}^{2}$ is plotted in Fig. 5(a), and compared with the value needed to fit data from HERMES. The backward extrapolation of the KN fit with $b_{\max }=1.5 \mathrm{GeV}^{-1}$ falls well below the value for the HERMES data. The BLNY fit with $b_{\max }=0.5 \mathrm{GeV}^{-1}$ even gives an unphysical reversed sign. TMD evolution at HERMES energies is evidently slower than is seen in the higher energy DY data.

But the $\tilde{K}\left(b_{\mathrm{T}}\right)$ function is universal. A clue to reconciling the different rates of evolution is in the table of typical values of $b_{\mathrm{T}}$ in Fig. $5(\mathrm{~b})$. We deduce that, because the BLNY and KN fits use higher-energy data, they determine $\tilde{K}\left(b_{\mathrm{T}}\right)$ primarily at lower $b_{\mathrm{T}}$ than is needed for HERMES data. So perhaps the extrapolation of $\tilde{K}\left(b_{\mathrm{T}}\right)$ to higher $b_{\mathrm{T}}$ is wrong, and its functional dependence flattens. If this conjecture is correct, then we can simultaneously fit all the data without violating factorization.

\section{A tool to compare different methods and fits: The $A$ function}

The work described in this section is from a forthcoming paper in collaboration with Ted Rogers. 


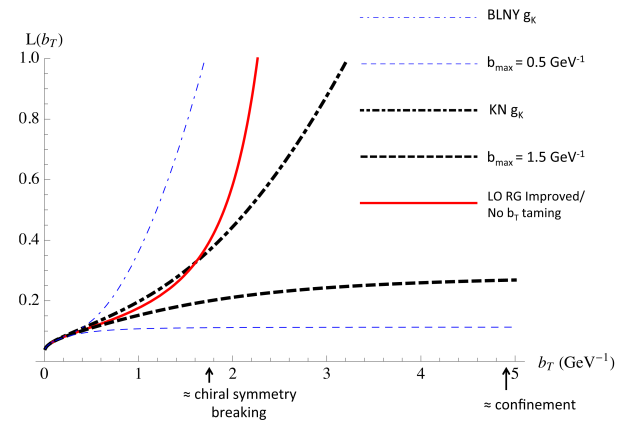

(a)

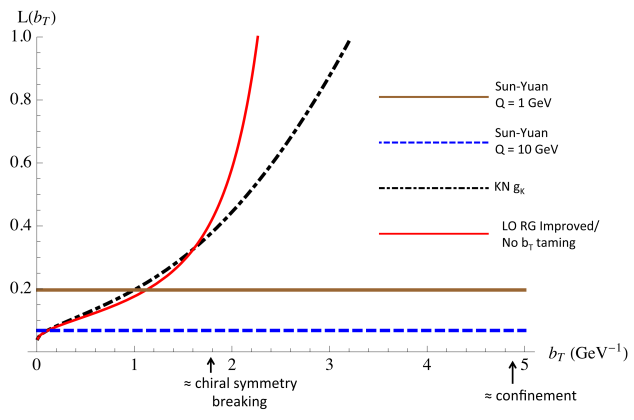

(b)

Fig. 6. Plots of $A\left(b_{\mathrm{T}}\right)$ : (a) for BLNY and KN, and their components; (b) for the Sun-Yuan ${ }^{6}$ analysis. The function is labeled $L$ here, instead of $A$.

A complication in comparing the different methods of working with TMD functions is that the evolution kernel is scheme and RG dependent. However, observe that the change of shape of the transverse momentum distribution comes only from $b_{\mathrm{T}}$-dependence of $\tilde{K}$. So we define the scheme-independent quantity ${ }^{\mathrm{a}}$

$$
A\left(b_{\mathrm{T}}\right)=-\frac{\partial}{\partial \ln b_{\mathrm{T}}^{2}} \frac{\partial}{\partial \ln Q^{2}} \ln \tilde{W}\left(b_{\mathrm{T}}, Q, x_{A}, x_{B}\right) \stackrel{\mathrm{CSS}}{=}-\frac{\partial}{\partial \ln b_{\mathrm{T}}^{2}} \tilde{K}\left(b_{\mathrm{T}}, \mu\right) .
$$

It is defined in any formalism where the cross section is the Fourier transform of a $b_{\mathrm{T}}$-space quantity. QCD predicts that, when factorization is valid, $A$ is (a) independent of $Q, x_{A}, x_{B}$, of light-quark flavor, (b) RG invariant, (c) perturbatively calculable at small $b_{\mathrm{T}}$. Like $\tilde{K}$ it is non-perturbative at large $b_{\mathrm{T}}$. We propose that $A\left(b_{\mathrm{T}}\right)$ should be presented for any available fits and calculations; differences give diagnostics as to where the different formalisms and fits can be tested.

In Fig. 6 are shown the first results of such comparisons. In both graphs, the red solid line is the RG-improved perturbative prediction for $A$; it, of course, diverges at the Landau pole. In graph (a), the thin blue dashed line is the cut-off perturbative part, with $b_{\max }=0.5 \mathrm{GeV}^{-1}$, and the thin blue dot-dashed line is the result of adding in the BLNY fit for $g_{K}$. The thick black dashed line and the thick black dot-dashed line are the corresponding curves for the $\mathrm{KN}$ fit with $b_{\max }=1.5 \mathrm{GeV}^{-1}$. Evidently the fit roughly reproduces the perturbative calculation somewhat beyond where it is turned down by the $b_{\max }$ cut off.

In graph (b) are again shown the pure RG-improved result and the full KN result for $A$, but now in comparison with values used by Sun and Yuan. ${ }^{6}$ They make an approximation to full TMD factorization, and from this one can easily calculate that their value for $A$ is $A_{\mathrm{SY}}=C_{F} \alpha_{s}(Q) / \pi$. It depends on $Q$, which is contrary to the QCD prediction. It is also independent of $b_{\mathrm{T}}$, which, while good

a The function was called $L$ in the talk. But the name $A$ is better, because it is essentially the same as a function of the same name defined by $\mathrm{CSS}^{4}$ 


\section{J. Collins}

for the desired slowing of TMD evolution at large $b_{\mathrm{T}}$, is also in contradiction with the unambiguous QCD predictions for small $b_{\mathrm{T}}$. In graph (b), the upper brown and lower blue horizontal lines give $A_{\mathrm{SY}}$ for $Q=1 \mathrm{GeV}$ and $Q=10 \mathrm{GeV}$. The flatness and relatively small value of $A_{\mathrm{SY}}$ at large $b_{\mathrm{T}}$ are what enable the HERMES data to be reproduced, while the rough equality with the value of $A$ at around $b_{\mathrm{T}}=1.5 \mathrm{GeV}^{-1}$ for the $\mathrm{KN}$ fit and for the perturbative calculation is presumably what also allows Sun and Yuan to reproduce the fixed-target DY data. But their form for $A$ is not consistent with the QCD prediction and the established fits at lower $b_{\mathrm{T}}$.

The above remarks indicate the nature of the modifications of functional form that one should make to the KN parameterization to enable one to preserve the good fit to DY data (and the corresponding measurement of $A$ ), while also having slower evolution at larger values of $b_{\mathrm{T}}$, as needed for the HERMES and COMPASS data. The results of a fit will have an important effect on predictions for the Sivers asymmetry at proposed polarized DY experiments.

\section{Implications}

It is important to determine the actual non-perturbative part of TMD evolution (i.e., $\tilde{K}\left(b_{\mathrm{T}}\right)$ at large $\left.b_{\mathrm{T}}\right)$. Older fits (e.g., KN) are satisfactory for $b_{\mathrm{T}}$ up to around $3 \mathrm{GeV}^{-1}=0.6 \mathrm{fm}$, or perhaps not even that far. But their extrapolation to larger $b_{\mathrm{T}}$ makes $\tilde{K}\left(b_{\mathrm{T}}\right)$ too large. The function $A\left(b_{\mathrm{T}}\right)$ can be used to diagnose the issues: It is a universal scheme independent function in QCD.

\section{Acknowledgments}

This work was supported by the U.S. Department of Energy. I thank many colleagues for discussions, notably Ted Rogers and Ahmad Idilbi.

\section{References}

1. F. Landry, R. Brock, P. M. Nadolsky and C.-P. Yuan, Phys. Rev. D67, p. 073016 (2003).

2. J. C. Collins, Foundations of Perturbative QCD (Cambridge University Press, Cambridge, 2011).

3. J. Collins and J.-W. Qiu, Phys. Rev. D75, p. 114014 (2007).

4. J. C. Collins, D. E. Soper and G. Sterman, Nucl. Phys. B250, 199 (1985).

5. P. Sun and F. Yuan, Phys. Rev. D88, p. 034016 (2013).

6. P. Sun and F. Yuan, Phys. Rev. D88, p. 114012 (2013).

7. M. G. Echevarría, A. Idilbi, A. Schäfer and I. Scimemi, Eur. Phys. J. C73, p. 2636 (2013).

8. G. Bozzi, S. Catani, D. de Florian and M. Grazzini, Nucl. Phys. B737, 73 (2006).

9. J. Collins arXiv:1409.5408.

10. A. V. Konychev and P. M. Nadolsky, Phys. Lett. B633, 710 (2006). 